\title{
Drag Reduction by Bubble-Covered Surfaces Found in PDMS Microchannel through Depressurization
}

\author{
Yang Gao ${ }^{1}$, Jiang $\mathrm{Li}^{2}$, Ho Cheung Shum ${ }^{3}$, Haosheng Chen *1 \\ ${ }^{1}$ State Key Laboratory of Tribology, Tsinghua University, Beijing, 100084, China \\ ${ }^{2}$ Mechanical engineering school, University of Science and Technology Beijing, \\ Beijing, 100083, China \\ ${ }^{3}$ Department of Mechanical Engineering, University of Hong Kong, Pokfulam Road, \\ Hong Kong \\ *Email:chenhs@tsinghua.edu.cn
}




\section{Supplemental Materials A}

Calculation of the local pressure and the theoretical flow rate. The pressure drop $\left(\Delta p_{\text {total }}\right)$ along the rectangular microchannel (Part b) and the cylindrical tubings (Part a and Part c) in the experiment can be expressed by equation (A1)-equation (A4) according to the Poiseuille flow equation:

$\Delta p_{a}=k_{1} Q \cdot L_{a} / R_{a}^{4}$

$\Delta p_{b}=k_{2} Q \cdot L_{b} / W h^{3}$

$\Delta p_{c}=k_{l} Q \cdot L_{c} / R^{4}$

$\Delta p_{\text {total }}=\Delta p_{a}+\Delta p_{b}+\Delta p_{c}$

Where $Q$ is the flow rate of the liquid, $k_{l}=8 \mu / \pi, k_{2}=12 \mu /(1-0.63 h / \mathrm{W}), \mu$ is the viscosity of the liquid, $L$ is the length of the microchannel or the cylindrical tubings, $R$ is the radius of the tubings, and $L_{a}=60 \mathrm{~cm}, R_{a}=100 \mu \mathrm{m}, W=100 \mu \mathrm{m}, h=40 \mu \mathrm{m}, L_{b}=1 \mathrm{~cm}, L_{c}=22.5 \mathrm{~cm}, R_{c}=190 \mu \mathrm{m}$. So the local pressure of the measurement point can be calculated to be $41.2 \%$ of the $\Delta p_{\text {total }}$. Once the pressure drop along the microchannel is calculated, the theoretical flow rate can be calculated according to equation (A5):

$Q=\Delta p_{a} \cdot R_{a}^{4} / k_{1} L_{a}$

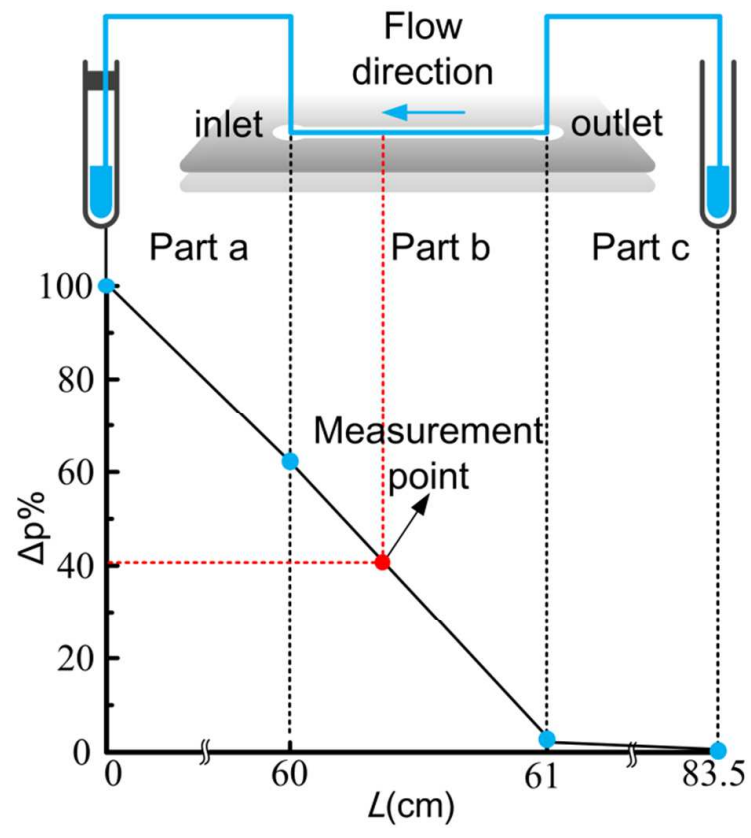

Figure A: The pressure drop along the experiment system. 


\section{Supplemental Materials B}

\section{The deformation of the PDMS channel under pressurization and depressurization condition.}

From 1000 mbar to 1400 mbar, the change in channel width as a result of deformation was less than $1.2 \mu \mathrm{m}$ for both pressurization and depressurization condition, which would lead to a change of less than $1.2 \%$ in the flow rate. Moreover, the reduction in channel width under depressurization condition would result in a decrease in the flow rate, contrary to the increase observed experimentally. For example, with a pressure of $900 \mathrm{mbar}$, for the observed drag reduction of $11.47 \%$, the channel width would have increased by $11.47 \mu \mathrm{m}$ according to the Poiseuille's Law in a rectangular channel, assuming change in channel width is the only factor causing the drag reduction. However, experimentally, the channel width decreased by $0.62 \mu \mathrm{m}$, as shown in Figure B below. This confirms that the drag reduction was not caused by the deformation of the channel.

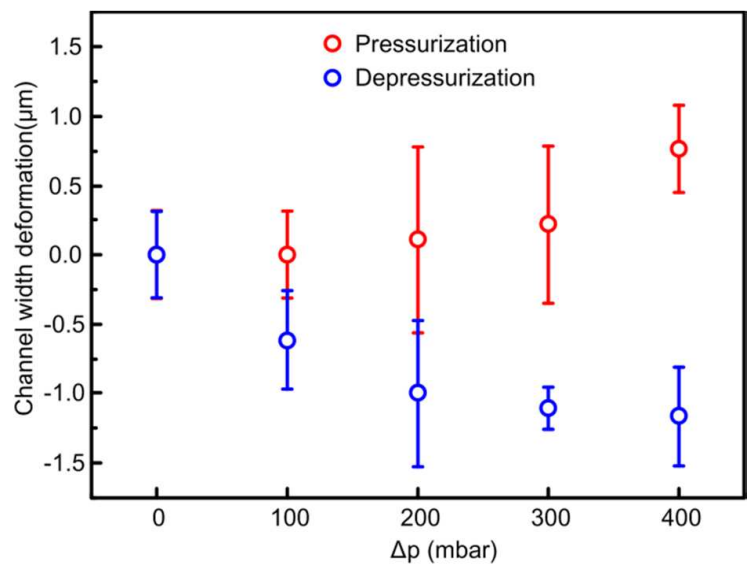

Figure B: Change in channel width due to deformation as a function of the applied pressure. The red symbols and the blue symbols represent the deformation of the channel under pressurization and depressurization condition, respectively. 


\section{Supplemental Materials C}

The flow rates measurement by controlling both the inlet and outlet pressures. We performed the experiment to control both the inlet and outlet pressures, as shown in Fig. C. For the flow direction 1, as illustrated by the red arrows, the pressure at the point $\mathrm{B}$ was fixed to $100 \mathrm{mbar}$, and the pressure difference was changed by changing the pressure at the point A. Similarly, for the flow direction 2, as illustrated by the blue arrows, the pressure at the point A was fixed to 100 mbar, and the pressure difference was changed by changing the pressure at the point $\mathrm{B}$. We found that the flow rates are the same for both the two flow directions at the same pressure difference. Thus, we think this system is symmetric and the drag reduction comes from the bubble-covered surface formed under reduced pressures.

(a)
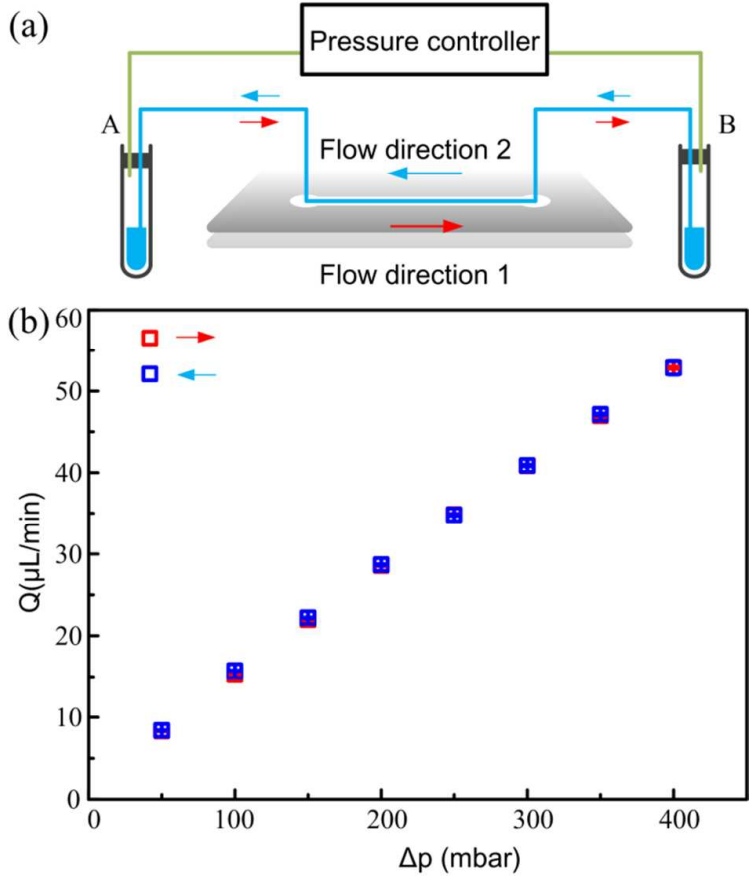

Figure C: (a) The schematic of flow rates measurement by controlling both the inlet and outlet pressures. (b) Average flow rates for the two flow directions at different pressure difference. 


\section{Supplemental Materials D}

The schematic of the AFM system for characterizing the PDMS surface in water under the depressurization condition. In the experiments under different reduced pressure, there was no change in the test position for studying the bubble growth.

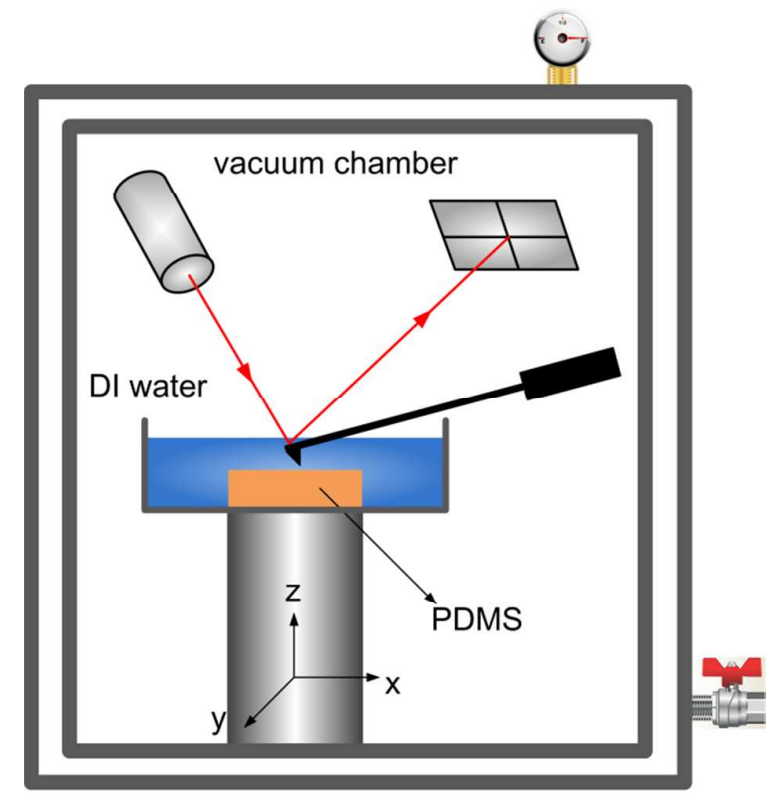

Figure D: The AFM measurement system under depressurization condition. The whole AFM system was placed inside the vacuum chamber in which the environmental pressure was controlled. The reduced pressure was supplied by a vacuum pump connected to the chamber. Both the PDMS and the scanning probe were immersed in water. 


\section{Supplemental Materials E}

AFM testing experiments. Considering the impact of ambient pressure on AFM measurement, the experiments have been done to measure the profile of the standard grating in water, as shown in Fig. E. This measurement shows that the process of pumping gas from the vacuum chamber may have a small impact on the AFM measurement. But as the AFM testing images shown, AFM can work properly for different ambient pressures.
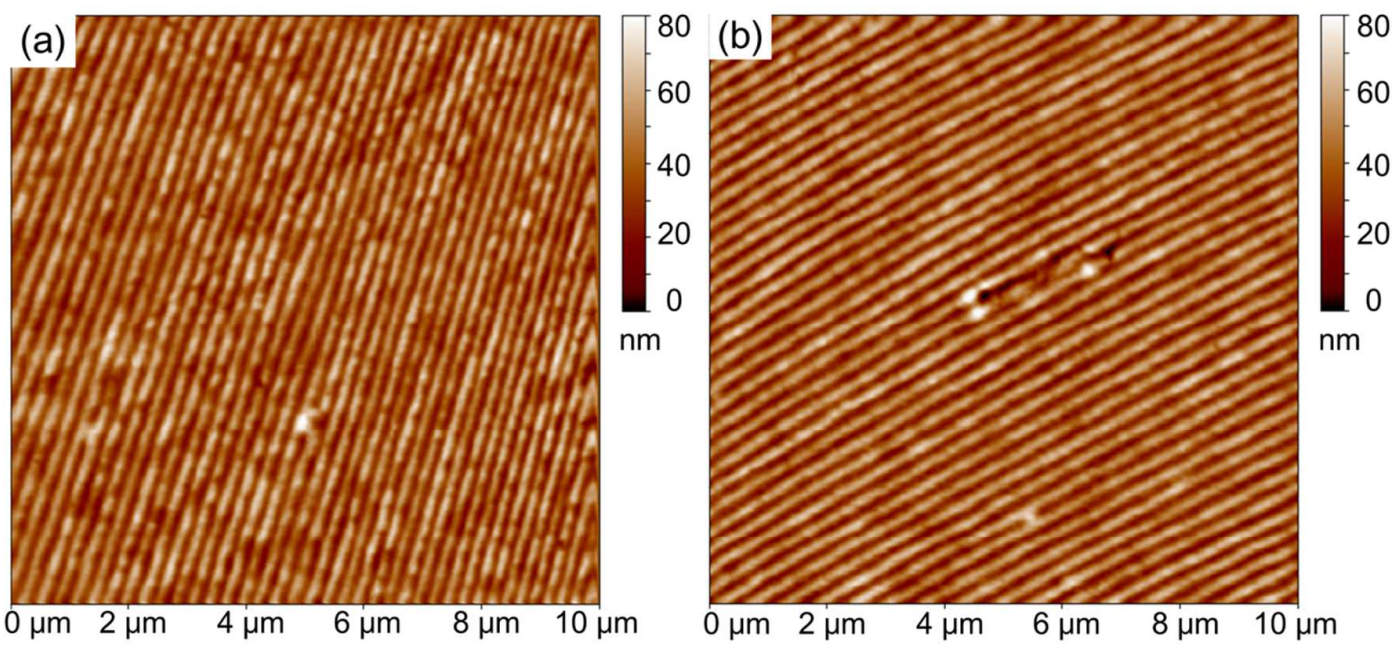

Figure E: (a) A $10 \times 10 \mu \mathrm{m}^{2}$ AFM tapping mode image of standard grating at the pressure of 1000 mbar in water. (b) A $10 \times 10 \mu \mathrm{m}^{2}$ AFM tapping mode image of standard grating at the pressure of $600 \mathrm{mbar}$ in water. 


\section{Supplemental Materials F}

The AFM image of dry PDMS substrate. The area roughness of the PDMS substrate: $\mathrm{Sa}=3.3$ nm.

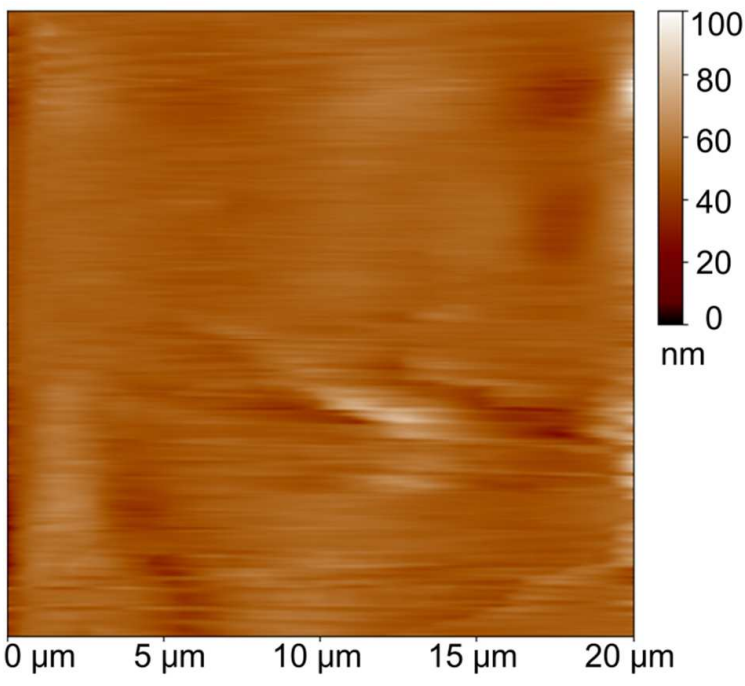

Figure F: A $20 \times 20 \mu \mathrm{m}^{2}$ AFM tapping mode image of the dry PDMS substrate. 


\section{Supplemental Materials G}

The statistical analysis of the protrusion size. The analysis is based on Fig. 3 to quantify the diameter of the protrusions.

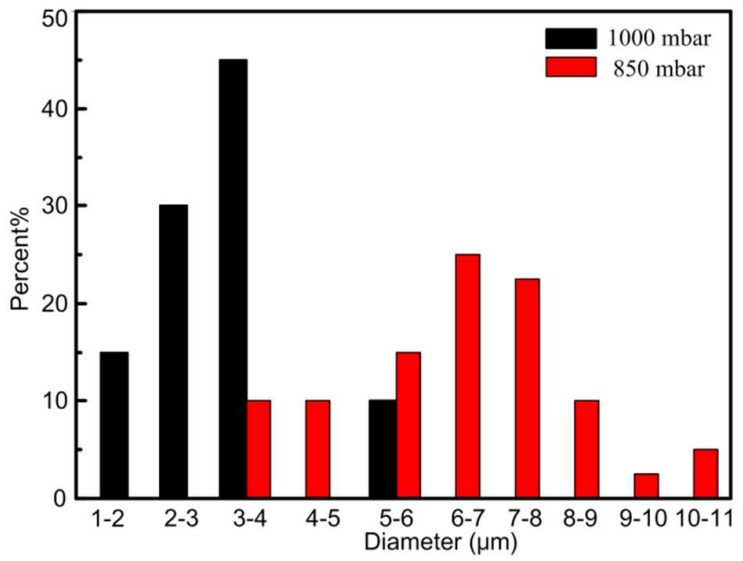

Figure G: The diameter distribution of the protrusion at different pressure. Sixty bubbles were measured. 


\section{Supplemental Materials H}

Gas content and flow rates measurement for DI water and degassed water. The three groups of degassed water were degassed with the same process, and their oxygen content was measured by a FireSting oxygen sensor. Assuming the proportion of oxygen in the dissolved gas is fixed, we can use the change of oxygen content to quantify the gas content. The measurement was performed at $25^{\circ} \mathrm{C}, 1000$ mbar. Using the degassed water, the average flow rates were calculated with flow rates values in three devices, and similar flow rates and drag reduction has been found for degassed water, as shown in Fig. H(b).
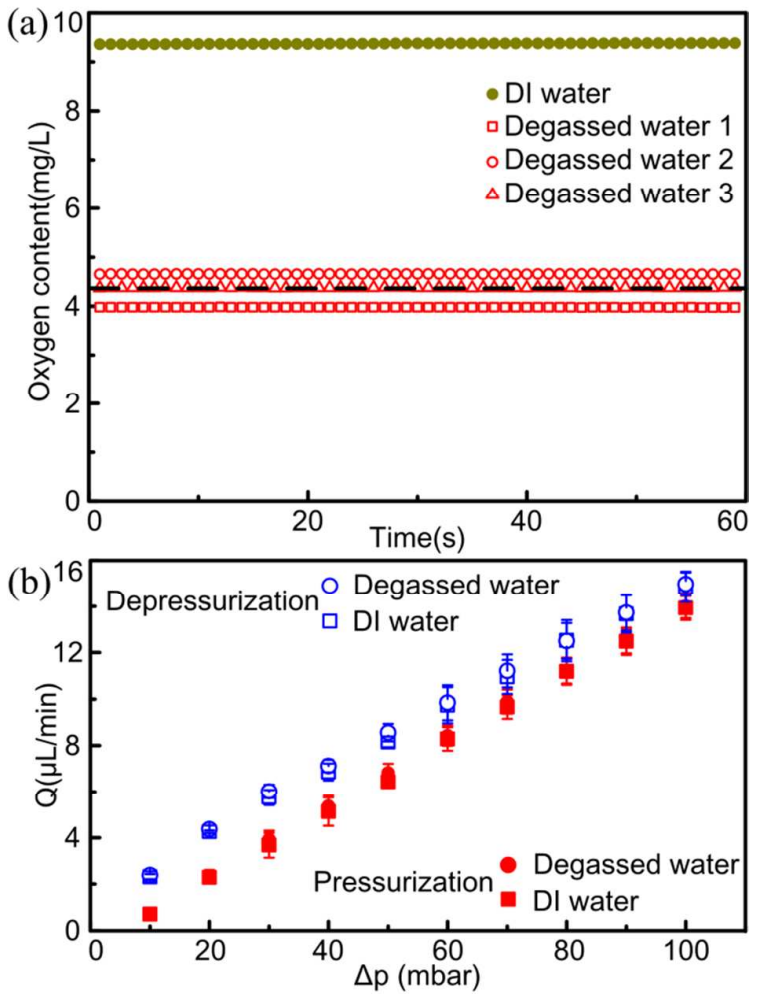

Figure H: (a) The oxygen content of DI water and degassed water. The dashed line shows the average oxygen content value calculated from the three degassed water groups. (b) The average flow rates comparison between the DI water and the degassed water with the gas content of $11.6 \mathrm{mg} / \mathrm{L}$, with $46 \%$ of saturation. The red and the blue symbols represent fluid under pressurization and depressurization-driven condition, respectively. 